Pacific Journal of Mathematics

GLOBAL REFLECTION FOR A CLASS OF SIMPLE CLOSED 


\section{GLOBAL REFLECTION FOR A CLASS OF SIMPLE CLOSED CURVES}

\section{JAMES M. SLOSS}

Global reflection is considered for a class of closed Jordan curves $\Gamma:[x(\theta), y(\theta)], 0 \leqq \theta<2 \pi$ where $x(\theta)$ and $y(\theta)$ are trigonometric polynomials. Every curve of this form is algebraic and global reflection across it reduces to investigating an algebraic function and its critical points. The reflection function is picked to be that solution of the algebraic equation that maps $\Gamma:[x(\theta), y(\theta)]$ pointwise into $[x(\theta),-y(\theta)]$. This function is defined and analytic except on a finite set of points inside $\Gamma$, and at each of these points it is continuous.

1. Introduction. Reflection across an analytic arc which is a generalization of inversion in a circle and reflection across a straight line, goes back to Schwarz. Because of the current interest, see e.g. [1], [3], [4], [5], in reflection of solutions of plane elliptic differential equations across analytic arcs, it seems appropriate to analyze the global reflection across a fairly general class of closed Jordan curves.

We shall investigate the class of Jordan rectifiable curves $\Gamma$ of the form

$$
\begin{aligned}
& x(\theta)=\sum_{k=0}^{n} a_{k} \cos k \theta+b_{k} \sin k \theta \\
& 0 \leqq \theta<2 \pi, n \geqq m
\end{aligned}
$$

with $x^{\prime 2}(\theta)+y^{\prime 2}(\theta) \neq 0,\left(a_{n}, b_{n}\right) \neq(0,0) \neq\left(\alpha_{m}, \beta_{m}\right)$ and if $m=n$ then either $\alpha_{n}^{2}+\beta_{n}^{2} \neq a_{n}^{2}+b_{n}^{2}$ or $\alpha_{n} a_{n}+\beta_{n} b_{n} \neq 0$.

The investigation will be reduced to analyzing a certain algebraic equation $M[z, \zeta]=0$ arising from (1.1) and (1.2) (see (2.5)-(2.8)).

Let $R$ be a simply-connected region bounded by a curve $\Gamma$ of the form (1.1) and (1.2). Let $S$ be the finite set of points made up of zeros of the resultant polynomials

$$
P(z)=R\left[M[z, \zeta], M_{\zeta}[z, \zeta]\right]=0
$$

and

$$
Q(z)=R\left[M[z, \zeta], M_{z}[z, \zeta]\right]=0 .
$$

Let $L_{i}$ be a rectifiable Jordan arc in $R$ containing $\left\{e_{1}, \cdots, e_{s}\right\}=R \cap S$. Then for one of the functions 


$$
\zeta=G(z)
$$

defined by $M[z, \zeta]=0$ we have

$1 G(z)$ is defined and analytic on $R-\left\{e_{1}, \cdots, e_{s}\right\}$,

$2 G(z)$ is single-valued on $R \backslash L_{i} \cup \Gamma$,

$3 \quad G^{\prime}(z) \neq 0$ on $R \backslash L_{i} \cup \Gamma$.

4 For $z$ on $\Gamma, \bar{z}=G(z)$.

$5 \overline{G\left[R \backslash L_{i}\right]} \cap R \backslash L_{i}=\varnothing$.

6 About each $e_{j}(1 \leqq j \leqq s)$ we have either

(i) $G(z)$ is defined, single-valued and analytic on a neighborhood of $e_{j}$ with

$$
G(z)=\left(z-e_{j}\right)^{2} G^{*}(z),
$$

$G^{*}(z)$ analytic and thus $G^{\prime}\left(e_{j}\right)=0$ or

(ii) on some neighborhood of $e_{j}$

$$
G(z)=\sum_{k=0}^{\infty} f_{k}\left[\sqrt[p]{z-e_{j}}\right]^{k}, \quad 1 \leqq p \leqq 2 n \quad(n \text { of }(1.1)),
$$

$f_{k}$ constant, or

(iii) $G(z)$ is defined, single-valued and analytic on a neighborhood of $e_{j}$ and $G^{\prime}\left(e_{j}\right) \neq 0$.

In the event $M[z, \zeta]$ is irreducible 6(iii) is excluded. We shall denote for $z$ in $R \backslash L_{i}$

$$
\widehat{z}=\overline{G(z)} .
$$

$G(z)$ is the reflection function and $\widehat{z}$ is the reflection of $z$ across $\Gamma$.

$7 G(z)$ can be extended to be defined and analytic and singlevalued on

$$
\left\{R \backslash L_{i}\right\} \cup \Gamma \cup\left\{\overline{G\left(R \backslash L_{i}\right)}\right\}=\left\{R \backslash L_{i}\right\} \cup \Gamma \cup\left\{\widehat{R \backslash L_{i}}\right\}
$$

with

$$
\widehat{\hat{z}}=z \text { for } z \text { in }\left\{R \backslash L_{i}\right\} \cup \Gamma \cup\left\{\widehat{R \backslash L}_{i}\right\} .
$$

It is the proof of 6(ii) that gives the most difficulty.

2. Geometrical reflection. To begin our investigation of reflection across a rectifiable Jordan curve $\Gamma$ of the form

$$
\begin{aligned}
& x(\theta)=\sum_{k=0}^{n} a_{k} \cos k \theta+b_{k} \sin k \theta \\
& y(\theta)=\sum_{k=0}^{m} \alpha_{k} \cos k \theta+\beta_{k} \sin k \theta
\end{aligned}
$$

we let 


$$
t=e^{i \theta}=\cos \theta+i \sin \theta
$$

and express (2.1) and (2.2) in terms of $t$ and $\bar{t}$, then (2.1) and (2.2) become:

$$
2 x=2 a_{0}+\bar{c}_{1} t+c_{1} \bar{t}+\bar{c}_{2} t^{2}+c_{2} \bar{t}^{2}+\cdots+\bar{c}_{n} t^{n}+c_{n} \bar{t}^{n}
$$

and

$$
2 y=2 \alpha_{0}+\bar{\gamma}_{1} t+\gamma_{1} \bar{t}+\bar{\gamma}_{2} t^{2}+\gamma_{2} \bar{t}^{2}+\cdots+\bar{\gamma}_{m} t^{m}+\gamma_{m} \bar{t}^{m}
$$

with

$$
c_{k}=a_{k}+i b_{k}, \quad \gamma_{k}=\alpha_{k}+i \beta_{k} .
$$

If we multiply (2.3) by $\bar{t}^{n}$ and (2.4) by $\bar{t}^{m}$ we see that (2.3) and (2.4) are equivalent respectively to:

$$
f(t) \equiv \bar{c}_{n}+\bar{c}_{n-1} \bar{t}+\cdots+\bar{c}_{1} \bar{t}^{n-1}+2\left(a_{0}-x\right) \bar{t}^{n}+c_{1} \bar{t}^{n+1}+\cdots+c_{n} \bar{t}^{2 n}=0
$$

and

$$
g(t) \equiv \bar{\gamma}_{m}+\bar{\gamma}_{m-1} \bar{t}+\cdots+\bar{\gamma}_{1} \bar{t}^{m-1}+2\left(\alpha_{0}-y\right) \bar{t}^{m}+\gamma_{1} \bar{t}^{m+1}+\cdots+\gamma_{m} \bar{t}^{2 m}=0 .
$$

Thus the curve $\Gamma$ is given by exactly those $t$ for which $f(t)=0$ and $g(t)=0$, i.e., by the common roots of $f(t)$ and $g(t)$. But a necessary and sufficient condition for $f(t)$ and $g(t)$ to have common roots is that Sylvester's determinant $D(f, g)$ of order $(2 n+2 m) \times(2 n+2 m)$ vanish. If we let

$$
\alpha(x)=2\left(a_{0}-x\right), \quad \beta(y)=2\left(\alpha_{0}-y\right)
$$

then

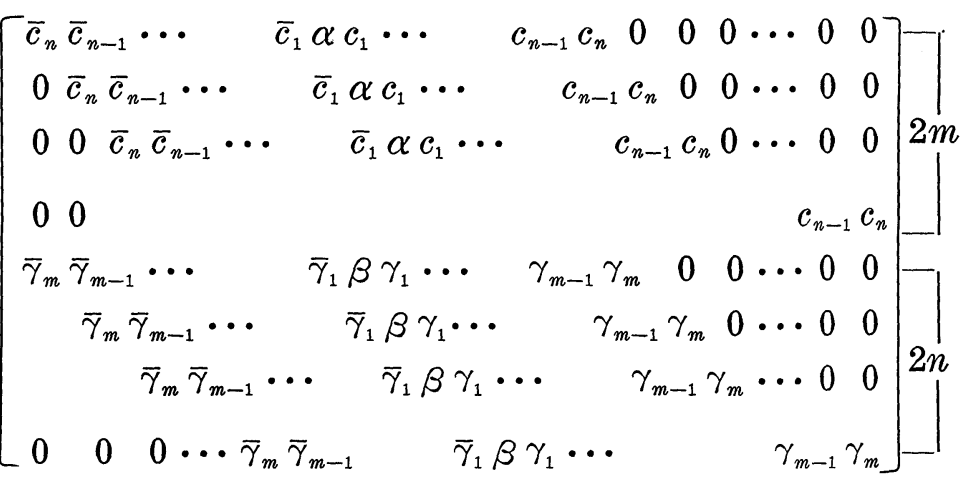

provided $c_{n} \neq 0 \neq \gamma_{m}$. Since $f$ and $g$ are fixed, we define

$$
\Delta[\alpha(x), \beta(y)]=D(f, g) \text {. }
$$

Then $\Gamma$ is given by the algebraic equation:

$$
\Delta[\alpha(x), \beta(y)]=0, \quad \alpha=2\left(a_{0}-x\right), \quad \beta=2\left(\alpha_{0}-y\right) .
$$


We now consider the algebraic equation of order $2 n$ in $\zeta$ :

$$
\begin{aligned}
M[z, \zeta] & =\Delta\left[\alpha\left(\frac{z+\zeta}{2}\right), \beta\left(\frac{z-\zeta}{2 i}\right)\right] \\
& =g_{2 n}(z) \zeta^{2 n}+g_{2 n-1}(z) \zeta^{2 n-1}+\cdots+g_{2}(z) \zeta^{2}+g_{1}(z) \zeta+g_{0}(z)=0
\end{aligned}
$$

and investigate the Riemann surface that this equation defines over the $z=x+i y$ plane.

First we prove:

LEMMA 2.1.

(i) If $n>m$ and $c_{n} \neq 0 \neq \gamma_{m}$, then

$$
g_{2 n}(z)= \pm \frac{1}{(2 n) !}\left|c_{n}\right|^{2 m}=\text { constant } \neq 0 .
$$

(ii) If $n=m$ and $c_{n} \neq 0 \neq \gamma_{n}$ and $c_{n}+i \gamma_{n} \neq 0 \neq \bar{c}_{n}+i \bar{\gamma}_{n}$, then

$$
g_{2 n}(z)= \pm \frac{1}{(2 n) !}\left(c_{n}+i \gamma_{n}\right)^{n}\left(\bar{c}_{n}+i \bar{\gamma}_{n}\right)^{n}=\text { constant } \neq 0 \text {. }
$$

Proof. First we note that

$$
g_{2 n}(z)=\frac{1}{(2 n) !} \frac{\partial^{2 n}}{\partial \zeta^{2 n}} M[z, \zeta]_{\zeta=0} .
$$

In order to differentiate $M[z, \zeta]$, it will be convenient to introduce the notation:

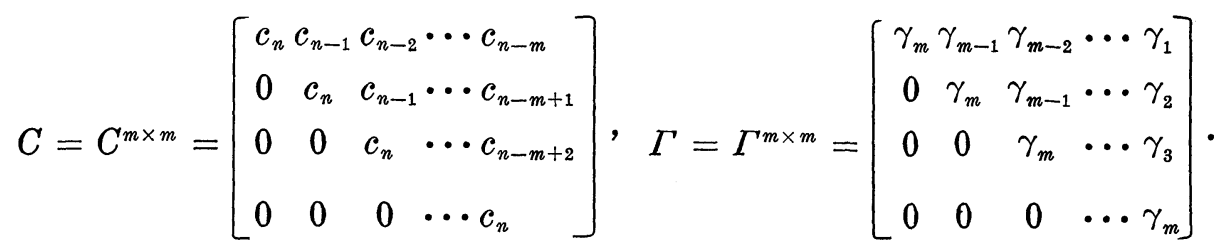

Then

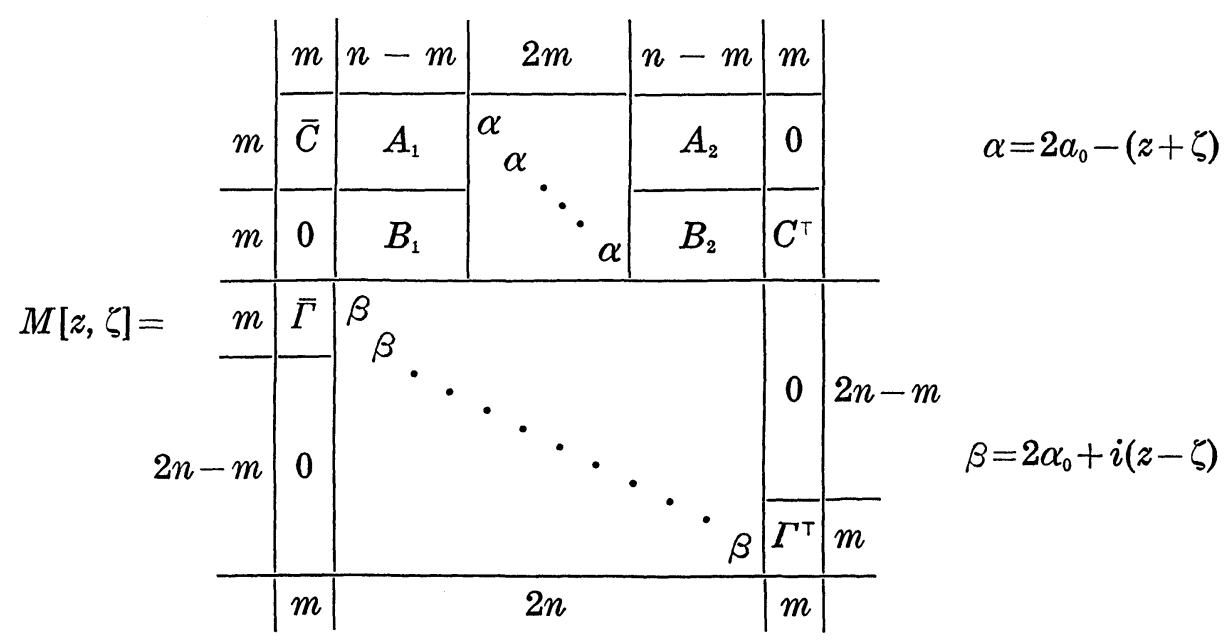


where, as indicated, $A_{1}, B_{1}, A_{2}, B_{2}$ are matrices of size $n-m \times m$. Thus

$$
\begin{aligned}
& \frac{\partial^{2 n}}{\partial \zeta^{2 n}} M[z, \zeta]=\begin{array}{|c|c|c|c|c|}
\hline \bar{C} & 0 & & 0 & 0 \\
\hline 0 & 0 & -I & 0 & C^{\top} \\
\hline \bar{\Gamma} & & & \\
\hline 0 & & -i I & & \\
\hline
\end{array}
\end{aligned}
$$

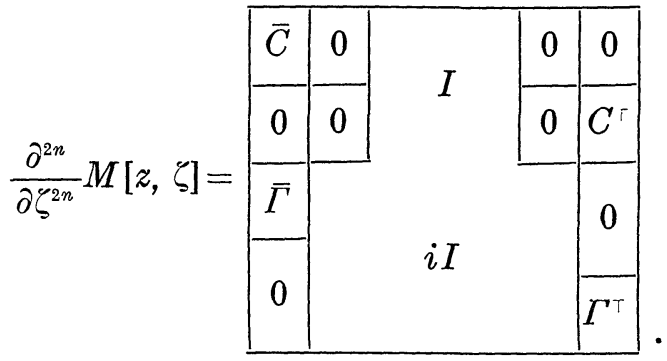

Next we perform the following set of operations

(i) multiply the $m+1$ column by $i \bar{\gamma}_{m}$ and add it to the first column

(ii) $)_{1}$ multiply the $m+1$ column by $i \bar{\gamma}_{m-1}$ and add it to the second column

(iii) $)_{1}$ multiply the $m+1$ column by $i \bar{\gamma}_{m-2}$ and add it to the third column etc. to $m$

(i ) $)_{2}$ multiply the $m+2$ column by $i \bar{\gamma}_{m}$ and add it to the second column

(ii) $)_{2}$ multiply the $m+2$ column by $i \bar{\gamma}_{m-1}$ and add it to the third column etc. to $m-1$

(i) $)_{m}$ multiply the $2 m$ column by $i \bar{\gamma}_{m}$ and add it to the $m$ th column. This yields the following result:

$$
\frac{\partial^{2 n}}{\partial \zeta^{2 n}} M[z, \zeta]=\begin{array}{|c|c|c|c|c|}
\hline \widetilde{C} & 0 & & 0 & 0 \\
\hline 0 & 0 & I & 0 & C^{\top} \\
\hline 0 & & & & 0 \\
\hline 0 & & i I & & \\
\hline
\end{array}
$$

where if $n-m \leqq m$, i.e., $n \leqq 2 m$ then 


$$
\widetilde{C}=\bar{C}+i\left(\begin{array}{cccccc}
n-m & & & & & \\
& \bar{\gamma}_{m} & \bar{\gamma}_{m-1} & \bar{\gamma}_{m-2} & \cdots & \\
0 & \bar{\gamma}_{m} & \bar{\gamma}_{m-1} & \cdots & \\
0 & 0 & \bar{\gamma}_{m} & \cdots & \\
0 & & & & & \\
& 0 & 0 & 0 & \cdots & 0 \\
& 0 & 0 & 0 & \cdots & 0
\end{array}\right)^{m \times m}
$$

and if $n-m>m$

$$
\widetilde{C}=\bar{C} .
$$

Next we perform the following set of operations

(i ) multiply column $2 n+m$ by $i \gamma_{m}$ and add it to column $2 n+2 m$

(ii) ${ }_{1}$ multiply column $2 n+m$ by $i \gamma_{m-1}$ and add it to column $2 n+2 m-1$

(iii) $)_{1}$ multiply column $2 n+m$ by $i \gamma_{m-2}$ and add it to column $2 n+2 m-2$ etc. to $m$

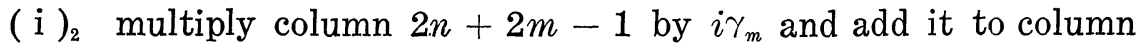
$2 n+2 m-1$

(ii) $)_{2}$ multiply column $2 n+m-1$ by $i \gamma_{m-1}$ and add it to column $2 n+2 m-2$ etc. to $m-1$

(i) $)_{m}$ multiply column $2 n+1$ by $i \gamma_{m}$ and add it to column $2 n+m+1$. This yields the following result:

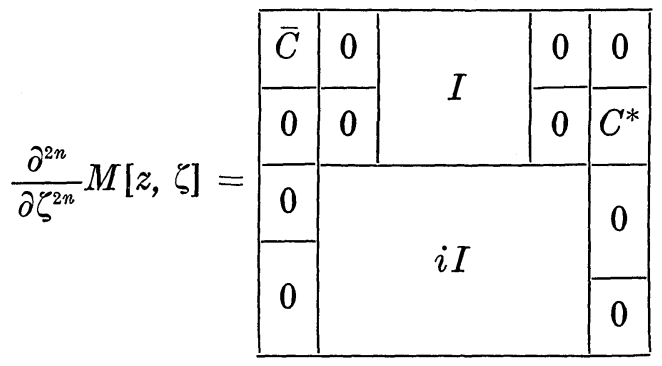

where if $n-m \leqq m$, i.e., $n \leqq 2 m$ then

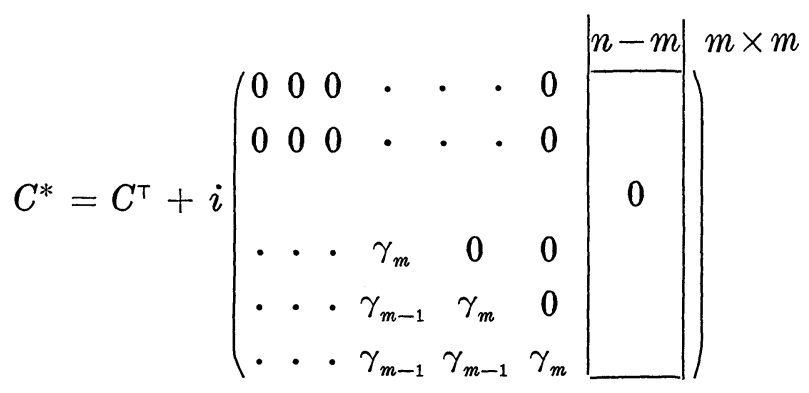


and if $n-m>m$

$$
C^{*}=C^{\top} .
$$

The next set of operations is as follows:

(i) multiply row $n+m+1$ by $i$ and add to row 1

(ii) multiply row $n+m+2$ by $i$ and add to row 2

(iii) multiply row $n+m+3$ by $i$ and add to row 3

(2m) multiply row $n+2 m$ by $i$ and add to row $2 m$. This yields:

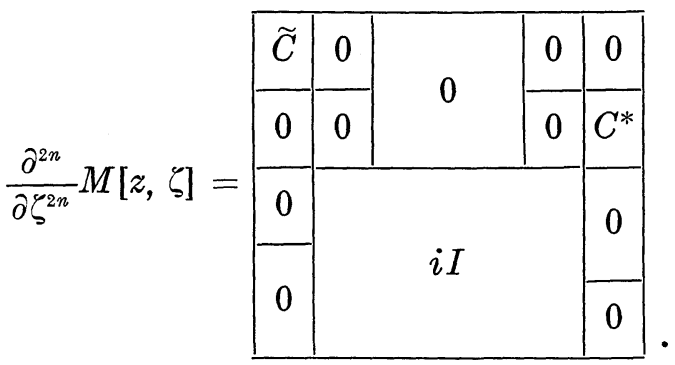

Since $c_{n} \neq 0$ and if $n=m, \bar{c}_{n}+i \gamma_{n} \neq 0 \neq c_{n}+i \gamma_{n}$ the above determinant, with appropriate column operations, is also given by:

$$
\begin{array}{c|c|c|c|c|c|}
\hline \partial^{2 n} & \widetilde{D} & 0 & & 0 & 0 \\
\hline 0 & 0 & 0 & 0 & D^{* 2 n} \\
\hline 0 & 0 & & & \\
\hline 0 & & I & & \\
\hline
\end{array}
$$

where

$$
\widetilde{D}=\begin{array}{ccc}
\bar{c}_{n} I^{m \times m} & \text { if } & n>m \\
\left(\bar{c}_{n}+i \bar{\gamma}_{n}\right) I^{n \times n} & \text { if } & n=m \\
c_{n} I^{m \times m} & \text { if } & n>m \\
D^{*}= & & \\
\left(c_{n}+i \gamma_{n}\right) I^{n \times n} & \text { if } & n=m .
\end{array}
$$

Thus

$$
\begin{aligned}
\frac{\partial^{2 n}}{\partial \zeta^{2 n}} M[z, \zeta] & = \pm\left(\bar{c}_{n}\right)^{m}\left(c_{n}\right)^{m} \quad \text { if } n>m \\
& = \pm\left(\bar{c}_{n}+i \bar{\gamma}_{n}\right)^{n}\left(c_{n}+i \gamma_{n}\right)^{n} \text { if } n=m
\end{aligned}
$$

which proves the lemma. 
If we write

$$
\begin{aligned}
M[z, \zeta] & =\Delta\left[\alpha\left(\frac{z+\zeta}{2}\right), \beta\left(\frac{z-\zeta}{2 i}\right)\right] \\
& =h_{2 n}(\zeta) z^{2 n}+h_{2 n-1}(\zeta) z^{2 n-1}+\cdots+h_{1}(\zeta) z+h_{0}(\zeta)=0,
\end{aligned}
$$

then we also have:

LEMMA 2.2 .

(i) If $n>m$ and $c_{n} \neq 0 \neq \gamma_{m}$ then

$$
h_{2 n}(\zeta)= \pm \frac{1}{(2 n) !}\left|c_{n}\right|^{2 m}=\text { constant } \neq 0 \text {. }
$$

(ii) If $n=m$ and $c_{n} \neq 0 \neq \gamma_{n}$ and $c_{n}-i \gamma \neq 0 \neq \bar{c}_{n}-i \bar{\gamma}_{n}$ then

$$
h_{2 n}(\zeta)= \pm \frac{1}{(2 n) !}\left(c_{n}-i \gamma_{n}\right)^{n}\left(\bar{c}_{n}-i \bar{\gamma}_{n}\right)^{n} \text {. }
$$

Proof. As in Lemma 2.1

$$
h_{2 n}(\zeta)=\frac{1}{(2 n) !} \frac{\partial^{2 n}}{\partial z^{2 n}} M[z, \zeta]_{z=0}
$$

and the proof proceeds as in Lemma 2.1.

We now recall some well-known facts from the theory of algebraic functions and Riemann surfaces, see e.g., [2].

We restrict ourselves to the case where $M[z, \zeta]$ is irreducible.

A point $z_{0}$ is called a critical point of $M[z, \zeta]$ if either

(i) $g_{2 n}\left(z_{0}\right)=0$ or

(ii) $M\left[z_{0}, \zeta\right]=0$ has multiple roots.

THEOREM (i). If $z_{0}$ is a point such that $g_{2 n}\left(z_{0}\right) \neq 0$ and $\zeta_{0}$ is a root of $M\left[z_{0}, \zeta\right]$ of multiplicity $l, 1 \leqq l \leqq 2 n$, then there exists an $\varepsilon>0$ and $\delta(\varepsilon)>0$ such that if $z_{1} \neq z_{0}$ lies in the disc $D\left(z_{0}, \delta\right)$ of radius $\delta$ about $z_{0}$ then $M\left(z_{1}, w\right)=0$ has exactly $l$ distinct roots in $D\left(\zeta_{0}, \varepsilon\right) . \quad[1]$, p. 122.

If $z_{0}$ is a point such that $g_{2 n}\left(z_{0}\right) \neq 0$ and $M\left[z_{0}, \zeta\right]$ has no multiple roots, $M\left[z_{0}, \zeta_{0}\right]=0$, then from the above theorem $l=1$ and for every $z=z_{1}$ in $D\left(z_{0}, \delta\right)$, there is exactly one root of $M\left[z_{1}, \zeta\right]=0$. Thus on $D\left(z_{0}, \delta\right) M[z, \zeta]=0$ defines a single-valued continuous function $\zeta=g_{1}(z)$ for which $\zeta_{0}=g_{1}\left(z_{0}\right)$.

THEOREM (ii). $g_{1}(z)$ is an analytic function of $z$ on $D\left(z_{0}, \delta\right)$.

In the case $g_{2 n}(z)=$ constant $\neq 0$ then there are at most a finite number of critical points. 
Let $e_{1}, e_{2}, \cdots, e_{r}$ be the critical points and join these and $\infty$ by $L_{0}$, any nonintersecting smooth arc and half line. Cut the plane along $L_{0}$. Then on the cut plane, $M[z, \zeta]=0$ defines $2 n$ single-valued analytic functions $G_{1}(z), \cdots, G_{2 n}(z)$.

THEOREM (iii). About each critical point e we have the following expansion in a neighborhood of $e$

$$
G(z)=\sum_{m=-\infty}^{\infty} f_{m}(\sqrt[p]{z-e})^{m}
$$

where

(1) $1 \leqq p \leqq 2 n$

(2) there are at most a finite number of negative powers

(3) if $g_{2 n}(e) \neq 0$ there are no negative powers.

We shall also need

\section{LEMMA 2.3. Let}

(1) $x^{\prime 2}(\theta)+y^{\prime 2}(\theta) \neq 0$

(2) $\left(a_{n}, b_{n}\right) \neq 0 \neq\left(\alpha_{n}, \beta_{n}\right)$

(3) either $\alpha_{n}^{2}+\beta_{n}^{2} \neq b_{n}^{2}+a_{n}^{2}$ or $\alpha_{n} a_{n}+b_{n} \beta_{n} \neq 0$

then for no point $z$ of $\Gamma$ (i) is $\zeta=\bar{z}$ a multiple root of $M[z, \zeta]$; (ii) is $g_{2 n}(z)=0$.

Proof. Since

$$
\begin{aligned}
& \left(\gamma_{n}-i c_{n}\right)=\left(\alpha_{n}+i \beta_{n}\right)-i\left(\alpha_{n}+i b_{n}\right)=\alpha_{n}+b_{n}+i\left(\beta_{n}-a_{n}\right) \\
& \left(\bar{\gamma}_{n}-i \bar{c}_{n}\right)=\left(\alpha_{n}-i \beta_{n}\right)-i\left(\alpha_{n}-i b_{n}\right)=\alpha_{n}-b_{n}-i\left(\beta_{n}+a_{n}\right)
\end{aligned}
$$

then

$$
\left(\gamma_{n}-i c_{n}\right)\left(\bar{\gamma}_{n}-i \bar{c}_{n}\right)=\alpha_{n}^{2}+\beta_{n}^{2}-\left(a_{n}^{2}+b_{n}^{2}\right)-2 i\left[\alpha_{n} a_{n}+b_{n} \beta_{n}\right] \neq 0 \text { by (2) . }
$$

Thus we see by Lemma 2.1 that $g_{2 n}\left(z_{0}\right)$ is never zero. For $z$ on $\Gamma$, i.e., for $z=x(\theta)+i y(\theta), 0 \leqq \theta<2 \pi$, we have

$$
M[x(\theta)+i y(\theta), x(\theta)-i y(\theta)] \equiv 0, \quad 0 \leqq \theta<2 \pi .
$$

Thus if we differentiate with respect to $\theta$, we see since (1) holds, that

$$
\frac{\left(x^{\prime}+i y^{\prime}\right)^{2}}{x^{\prime 2}+y^{\prime 2}}=\frac{M_{\zeta}[z, \bar{z}]}{M_{z}[z, \bar{z}]} \neq 0 \quad \text { on } \quad \Gamma
$$

Thus $M_{\zeta}\left[z_{0}, \zeta\right] \neq 0$ for $\zeta$ on $\Gamma$, i.e., for $\zeta=\bar{z}_{0}$ and the proof is complete.

Lemma 2.4. Let

(1) $\left(a_{n}, b_{n}\right) \neq 0 \neq\left(\alpha_{n}, \beta_{n}\right)$ and 
(2) either $\alpha_{n}^{2}+\beta_{n}^{2} \neq a_{n}^{2}+b_{n}^{2}$ or $\alpha_{n} a_{n}+\beta_{n} b_{n} \neq 0$ then there are at most a finite number of $z$ for which

$$
M[z, \zeta]=0 \text { and } M_{z}[z, \zeta]=0 \text {. }
$$

Proof. $z$ is a point at which the result of the lemma holds if and only if for $\zeta$ fixed

$$
M_{1}[z]=M[z, \zeta]
$$

has a multiple root. Thus

$$
\begin{aligned}
& M_{1}[z]=h_{2 n}(\zeta) z^{2 n}+h_{2 n-1}(\zeta) z^{2 n-1}+\cdots+h_{0}(\zeta) \\
& M_{1}^{\prime}[z]=2 n h_{2 n}(\zeta) z^{2 n-1}+\cdots+h_{1}(\zeta)
\end{aligned}
$$

and from Lemma 2.2 and the assumption of the lemma

$$
h_{2 n}(\zeta)=\text { constant } \neq 0 \text {. }
$$

But a necessary and sufficient condition for $M_{1}[z]$ to have a multiple root is that the resultant

$$
R\left[M_{1}, M_{1}^{\prime}\right]=0 .
$$

As this is a polynomial in $\zeta$, the conclusion of the lemma follows.

Lemma 2.5. Let the hypotheses of Lemma 2.3 hold, then for no point $z$ of $\Gamma$ do we have for $\zeta=\bar{z}$

$$
M[z, \zeta]=0 \text { and } M_{z}[z, \zeta]=0 .
$$

Proof. From the proof of Lemma 2.3

$$
\frac{M_{z}[z, \bar{z}]}{M_{\zeta}[z, \bar{z}]}=\frac{\left(x^{\prime}-i y^{\prime}\right)^{2}}{x^{\prime 2}+y^{\prime 2}} \neq 0
$$

which is the conclusion.

We shall assume that for $\Gamma$ we have

(1) $x^{\prime 2}(\theta)+y^{\prime 2}(\theta) \neq 0$

(2) $\left(a_{n}, b_{n}\right) \neq 0 \neq\left(\alpha_{n}, \beta_{n}\right)$

(3) either $\alpha_{n}^{2}+\beta_{n}^{2} \neq\left(b_{n}^{2}+a_{n}^{2}\right)$ or $\alpha_{n} a_{n}+b_{n} \beta_{n} \neq 0$.

Assume, moreover, that $M[z, \zeta]$ is irreducible. Let $e_{1}, e_{2}, \cdots, e_{r}$ be the set of critical points of $M[z, \zeta]=0$ and let $e_{r+1}, e_{r+2}, \cdots, e_{s_{0}}$ (by Lemma 2.4) be the set of $z$ for which

$$
M\left[e_{j}, \zeta\right]=0 \text { and } M_{z}\left[e_{j}, \zeta\right]=0
$$

and let

$$
S=\left\{e_{j}: 1 \leqq j \leqq s_{0}\right\}
$$


Let $z_{0}$ be a point of $\Gamma$ and $M\left[z_{0}, \bar{z}_{0}\right]=0$. By Lemma 2.3, $\zeta=\bar{z}_{0}$ is not a multiple root of $M\left[z_{0}, \zeta\right]$ and thus $M\left[z_{0}, \zeta\right]=0$ defines a singlevalued function of $z, \zeta=G(z)$ in some neighborhood $N_{0}$ of $z_{0}$ with $G\left(z_{0}\right)=\bar{z}_{0}$. Moreover, for each point $z$ on $\Gamma \cap N_{0}$ we have

$$
\bar{z}=G(z)
$$

by Lemma 2.3. Analytically continuing $G(z)$ around $\Gamma$ we return to $G(z)$. Since if we arrive at $G_{1}(z)$ where $G(z)$ and $G_{1}(z)$ are defined on a common neighborhood of $z_{0}$, then for $z$ on $\Gamma, z(\theta)=x(\theta)+i y(\theta)$ and $\zeta(\theta)=\overline{z(\theta)}$ is a periodic function and thus

$$
G(z)=G_{1}(z) \text { on } \Gamma \text {. }
$$

Therefore, they agree on the common neighborhood. From this it follows that $G(z)$ is single-valued and analytic on a neighborhood of $\Gamma$.

Let $S_{i}=\left\{e_{1}, \cdots, e_{s}\right\}$ be that subset of $S$ (renumber if necessary) that is contained in the interior of $\Gamma$ and let $S_{e}=S \backslash S_{i}$, then $G(z)$ is analytic on $R \backslash S_{i}$. Moreover, if we join each $e_{j}$ of $S_{i}$ by a Jordan $\operatorname{arc} L_{i}$ then $G(z)$ is analytic and single-valued on

$$
R_{0}=R \backslash L_{i} \cup \text { neighborhood of } \Gamma \text {. }
$$

Since on $R_{0}$ we have $G(z)$ is single-valued and analytic then

$$
G^{\prime}(z)=-\left.\frac{M_{z}[z, \zeta]}{M_{\zeta}[z, \zeta]}\right|_{\zeta=G(z)}
$$

which is $\neq 0$ for $z$ on $\Gamma$ and $\zeta=\bar{z}$ on $\Gamma$ by Lemma 2.5, and also $\neq 0$ for $z$ on $R \backslash L_{i}$ since all of the points $\left.M_{z}[z, \zeta]\right|_{\zeta=G(z)}=0$ lie on $L_{i}$ by construction. Thus we have partially proved the

THEOREM. $M[z, \zeta]=0$ defines a function

$$
\zeta=G(z)
$$

which is determined by having $z_{0}$ on $\Gamma$ correspond to $\bar{z}_{0}=G\left(z_{0}\right)$. For this $G(z)$ we have of $\Gamma$.

(1) $G(z)$ is defined and analytic on $R-\left\{e_{1}, \cdots e_{s}\right\} \cup$ neighborhood

(2) $G(z)$ is single-valued on $R \backslash L_{i} \cup$ neighborhood of $\Gamma$.

(3) $G^{\prime}(z) \neq 0$ on $R \backslash L_{i} \cup$ neighborhood of $\Gamma$.

(4) For $z$ on $\Gamma, \bar{z}=G(z)$.

(5) $\overline{G\left[R \backslash L_{i}\right]} \cap R \backslash L_{i}=\varnothing$.

(6) About each $e_{j}(1 \leqq j \leqq s)$ we have either

(i ) $G(z)$ is defined, single-valued and analytic on a neighborhood of $e_{j}$ with 


$$
G(z)=\left(z-e_{j}\right)^{2} G^{*}(z)
$$

$G^{*}(z)$ analytic and thus $G^{\prime}\left(e_{j}\right)=0$ or

(ii) on some neighborhood of $e_{j}$

$$
G(z)=\sum_{k=0}^{\infty} f_{k}\left[\sqrt[p]{z-e_{j}}\right]^{k}, \quad 1 \leqq p \leqq 2 n,
$$

$f_{k}$ constant, or

(7) If we let $\widehat{z}=G(z)$ then $G(z)$ can be extended to be defined analytic and single-valued on

with

(i) $\left\{R \backslash L_{i}\right\} \cup \Gamma \cup\left\{\widehat{R \backslash L_{i}}\right\}$

(ii) $\widehat{\hat{z}}=z$ there.

Proof. (1)-(4) have been proved.

(5) Since $\overline{G(\Gamma)}=\Gamma$ and since $G$ is continuous on $R \backslash L_{i}$ we know either $\overline{G\left[R \backslash L_{i}\right]} \subset R$ or $\overline{G\left[R \backslash L_{i}\right]} \cap R \backslash L_{i}=\varnothing$. We shall have proved the result if we can show that for one point $z \in R \backslash L_{i}, \overline{G(z)} \notin R$.

Since $x(\theta)$ and $y(\theta)$ are analytic functions for real $\theta$ with $x^{\prime 2}(\theta)+$ $y^{\prime 2}(\theta) \neq 0$ they can be continued as analytic functions $x(\tau)$ and $y(\tau)$ of the complex variable $\tau=\theta+i \eta$ on some circle $|\tau|<\rho$ for which, on $|\tau|<\rho, x^{\prime}(\tau)+i y^{\prime}(\tau) \neq 0$. Then

$$
g(\tau)=x(\tau)+i y(\tau) \quad|\tau|<\rho
$$

maps $\tau=\theta+i \eta, \eta=0$ onto a subarc $\Gamma_{0}$ of $\Gamma$ and thus maps $|\tau|<\rho$ 1-1 onto a neighborhood of $\Gamma_{0}$. Consider

$$
H(\tau)=\overline{G[g(\tau)]}
$$

for $\tau$ such that $g(\tau) \subset$ domain of $G$. Since $G(z)$ is defined and $G^{\prime}(z) \neq 0$ on a neighborhood of $\Gamma$ then $H(\tau)$ is defined on a neighborhood $N$ of $|\tau|\left\langle\rho, \eta=0\right.$ with $\eta=0$ mapping onto $\Gamma_{0}$ and $H(\tau)$ establishes a 1-1 correspondence between points of $N$ and $H(N)$. Thus that portion $N$ of $N$ for which $\eta<0$ maps onto the region $R_{-}$of one side of $\Gamma_{0}$ and $N_{+}$, that portion of $N$ for which $\eta>0$ maps onto the other side $R_{+}$of $\Gamma_{0}$. Without loss of generality let $R_{-} \cap R \backslash L_{i} \neq \varnothing$, $R_{+} \cap R \backslash L_{i}=\varnothing$ and let $z_{0} \in R_{-} \cap R \backslash L_{i}$ be such that if $g\left(\tau_{0}\right)=z_{0}$ then $\bar{\tau}_{0} \in N_{+}$. Then $g\left(\bar{\tau}_{0}\right) \in R_{+}$. Note that on that neighborhood of $\eta=0$ where everything is defined

$$
\overline{g^{-1}[\overline{G[g(\tau)]}]},
$$

is analytic with

$$
\overline{g^{-1}[\overline{G[g(\tau)]}]}=\overline{g^{-1}[g(\tau)]}=\bar{\tau}=\tau
$$

for $\tau$ on $\eta=0$ and thus is the identity map. Hence 


$$
g\left(\bar{\tau}_{0}\right)=\overline{G\left[g\left(\tau_{0}\right)\right]}=\overline{G\left[z_{0}\right]}
$$

and $\overline{G\left(z_{0}\right)} \in R_{+}$where as $z_{0} \in R \backslash L_{i}$. This completes the proof of (5). 2.1 .

(6) (i) and (ii) follow immediately from Theorem (iii) and Lemma

(7) (i) follows from (3) and (5). (ii) follows from the fact that

$$
\widehat{\widehat{z}}=\overline{G[\overline{G(z)}]}
$$

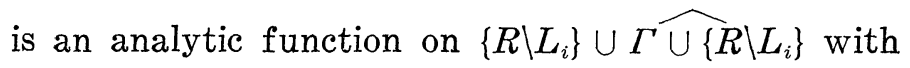

$$
\widehat{\widehat{z}}=z \quad \text { on } \Gamma
$$

and thus

$$
\widehat{\hat{z}}=z \quad \text { on } \quad\left\{R \backslash L_{i}\right\} \cup \Gamma \cup\left\{\widehat{R \backslash L_{i}}\right\}
$$

and the theorem is proved.

In the event $M[z, \zeta]$ is not irreducible then the analysis and the theorem will hold provided we decompose $M[z, \zeta]$ into its irreducible factors and (1) study that factor which determines $\Gamma$ and (2) prove that for this factor we have the coefficient of the highest order term in $\zeta$ is constant and the coefficient of the highest order term in $z$ is constant. We shall be possibly excluding an unnecessary number of points $e_{j}$ where $G(z)$ may be analytic single-valued and $G^{\prime}(z) \neq 0$. To see that the coefficient of the highest order term of $\zeta$ and $z$ are constants we let

$$
M[z, \zeta] \equiv Q_{1}(z, \zeta) Q_{2}(z, \zeta) \cdots Q_{r}(z, \zeta)
$$

where the $Q_{j}(z, \zeta)$ are irreducible. Then if

$$
Q_{j}(z, \zeta)=q_{s_{j}}(z) \zeta^{s_{j}}+q_{j_{s_{j}-1}}(z) \zeta^{s_{j}-1}+\cdots+q_{j 0}(z)
$$

we have $s_{1}+s_{2}+\cdots+s_{r}=2 n$. Moreover,

$$
q_{j s_{3}}(z)=\text { constant } \neq 0 \text { for all } j=1,2, \cdots, r,
$$

since

$$
q_{1 s_{1}}(z) \cdot q_{2 s_{2}}(z) \cdots q_{r s_{r}}(z) \equiv g_{2 n}(z)=\text { constant } .
$$

Similarly if we write

$$
Q_{j}(z, \zeta) \equiv p_{j s_{j}}(\zeta) z^{s_{j}}+p_{j_{s_{j}-1}}(\zeta) z^{s_{j}-1}+\cdots+p_{j 0}(\zeta)
$$

we see that

$$
p_{j s_{j}}(\zeta)=\text { constant } \neq 0 \text { for all } j=1,2, \cdots, r .
$$

It would be of interest to find conditions on the $c_{k}$ and $\gamma_{k}$ so 
that $M[z, \zeta]$ is irreducible. This would eliminate the calculation of an unnecessary number of points.

\section{REFERENCES}

1. P. R. Garabedian, Partial Differential Equations, John Wiley \& Sons, 1964, (see references).

2. K. Knopp, Theory of Functions, Part II. Transl. by F. Bagemihl, Dover Pub., 1949. 3. H. Lewy, On the reflection laws of second order differential equations on two independent variables, Bull. Amer. Math. Soc., 65 (1959), 37-58.

4. J. M. Sloss, Reflection laws of second order systems of elliptic equations in two independent variables with constant coefficients, Pacific J. Math., 24 (1968), 541-575. 5.- Reflection laws of high order elliptic differential equations in two independent variables with constant coefficients and unequal characteristics across analytic boundary conditions, Duke Math. J., 35 (1968), 415-434.

Received January 17, 1973.

University of California, Santa Barbara 


\section{PACIFIC JOURNAL OF MATHEMATICS}

EDITORS

RICHARD ARENS (Managing Editor)

University of California

Los Angeles, California 90024

R. A. Beaumont

University of Washington

Seattle, Washington 98105

J. DugundJI

Department of Mathematics

University of Southern California

Los Angeles, California 90007

D. Gilbarg and J. Milgram

Stanford University

Stanford, California 94305

\section{ASSOCIATE EDITORS}

E. F. BECKENBACH

B. H. NeumanN

F. WOLF

K. YOSHIDA

\section{SUPPORTING INSTITUTIONS}

UNIVERSITY OF BRITISH COLUMBIA CALIFORNIA INSTITUTE OF TECHNOLOGY

UNIVERSITY OF CALIFORNIA

MONTANA STATE UNIVERSITY

UNIVERSITY OF NEVADA

NEW MEXICO STATE UNIVERSITY

OREGON STATE UNIVERSITY

UNIVERSITY OF OREGON

OSAKA UNIVERSITY

\author{
UNIVERSITY OF SOUTHERN CALIFORNIA \\ STANFORD UNIVERSITY \\ UNIVERSITY OF TOKYO \\ UNIVERSITY OF UTAH \\ WASHINGTON STATE UNIVERSITY \\ UNIVERSITY OF WASHINGTON \\ $\stackrel{*}{*} \stackrel{*}{*}{ }^{*}{ }^{*}{ }^{2}$ AMERICAN MATHEMATICAL SOCIETY \\ NAVAL WEAPONS CENTER
}




\section{Pacific Journal of Mathematics}

\section{Vol. 52, No. $1 \quad$ January, 1974}

David R. Adams, On the exceptional sets for spaces of potentials ............ 1

Philip Bacon, Axioms for the Čech cohomology of paracompacta ............ 7

Selwyn Ross Caradus, Perturbation theory for generalized Fredholm operators ..... 11

Kuang-Ho Chen, Phragmén-Lindelöf type theorems for a system of nonhomogeneous equations ............................ 17

Frederick Knowles Dashiell, Jr., Isomorphism problems for the Baire classes .......

M. G. Deshpande and V. K. Deshpande, Rings whose proper homomorphic images are right subdirectly irreducible . . . . . . . . . . . . . . . . . . . . . . . . .

Mary Rodriguez Embry, Self adjoint strictly cyclic operator algebras .............

Paul Erdős, On the distribution of numbers of the form $\sigma(n) / n$ and on some related

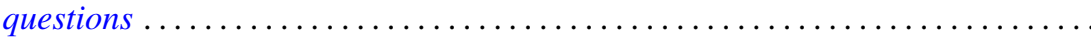

Richard Joseph Fleming and James E. Jamison, Hermitian and adjoint abelian

operators on certain Banach spaces ............................

Stanley P. Gudder and L. Haskins, The center of a poset .................. 85

Richard Howard Herman, Automorphism groups of operator algebras . . . ........

Worthen N. Hunsacker and Somashekhar Amrith Naimpally, Local compactness of families of continuous point-compact relations ....................

Donald Gordon James, On the normal subgroups of integral orthogonal groups ....

Eugene Carlyle Johnsen and Thomas Frederick Storer, Combinatorial structures in

loops. II. Commutative inverse property cyclic neofields of prime-power

order.

Ka-Sing Lau, Extreme operators on Choquet simplexes . . . . . . . . . . . . . . 129

Philip A. Leonard and Kenneth S. Williams, The septic character of 2, 3, 5 and $7 \ldots 143$

Dennis McGavran and Jingyal Pak, On the Nielsen number of a fiber map ........ 149

Stuart Edward Mills, Normed Köthe spaces as intermediate spaces of $L_{1}$ and

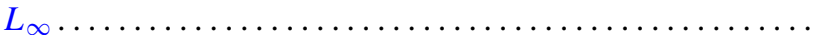

Philip Olin, Free products and elementary equivalence. .

Louis Jackson Ratliff, Jr., Locally quasi-unmixed Noetherian rings and ideals of the principal class.

Seiya Sasao, Homotopy types of spherical fibre spaces over spheres ...

Helga Schirmer, Fixed point sets of polyhedra ...

Kevin James Sharpe, Compatible topologies and continuous irreducible

representations.

Frank Siwiec, On defining a space by a weak base . . . . . . . . . . . . . . . 233

James McLean Sloss, Global reflection for a class of simple closed curves ....... 247

M. V. Subba Rao, On two congruences for primality . .

Raymond D. Terry, Oscillatory properties of a delay differential equation of even

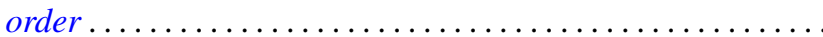

Joseph Dinneen Ward, Chebyshev centers in spaces of continuous functions . .

Robert Breckenridge Warfield, Jr., The uniqueness of elongations of Abelian

groups...

V. M. Warfield, Existence and adjoint theorems for linear stochastic differential

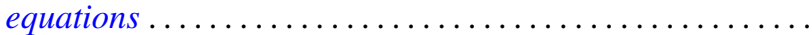

